\title{
Actitudes de docentes y estudiantes hacia la discapacidad en la Universidade da Coruña
}

\author{
Jessica Garabal-Barbeira \\ Universidade da Coruña
}

\begin{abstract}
Resumen
Objetivo: Determinar las actitudes de alumnos y docentes hacia los estudiantes con discapacidad. Metodología: Estudio descriptivo de prevalencia realizado en la UDC a docentes y alumnos, se ha utilizado la escala CUNIDIS. Resultados: Los participantes creen preciso adaptar los materiales utilizados en las aulas, pero no los criterios de evaluación y cualificación; indicaron que estas adaptaciones no se llevan a cabo realmente. La mayoría de los docentes reflejaron que no están formados para responder a las necesidades de los estudiantes con discapacidad. Conclusión: Docentes y estudiantes presentan, en general, unas actitudes favorables hacia la inclusión de los estudiantes con discapacidad.
\end{abstract}

Palabras clave: universidad, discapacidad, actitud, diversidad funcional.

La educación es un elemento fundamental para la integración social de toda persona, y por ello es un campo fundamental para las personas con discapacidad. Asegurar un sistema educativo lo más integrador posible debe ser uno de los pilares fundamentales de toda política estatal. El II Estudio sobre el grado de inclusión en el sistema universitario español respetando la realidad de la discapacidad manifiesta la existencia de 17702 alumnos con discapacidad (1,3\%), manifestando diferencias entre los que cursan estudios en modalidad presencial $(0,9 \%)$ y a distancia $(3,1 \%)$ (Fundación Universia, \& Cermi, 2013).

La actitud es una predisposición aprendida para responder consistentemente de un modo favorable o desfavorable ante personas o situaciones, así pues, es considerada como algo que se adquiere y, por tanto, que se puede modificar (Alonso Olea, Navarro, \& Vicente, 2007). Con respecto a esta variable la literatura existente incide en que el éxito de un sistema educativo inclusivo está directamente relacionado con las actitudes positivas de la comunidad educativa (Martínez, Bueno, \& Limiñana, 2011). Las actitudes constituyen uno de los principales elementos que pueden facilitar o dificultar el proceso de inclusión del alumnado con discapacidad en la educación superior y su conocimiento puede ayudar a propiciar un cambio de actitudes con el fin de lograr que las universidades reflejen una visión positiva de las personas con discapacidad, con posibilidades, potencialidades, capacidades, derechos y libertades equiparables a las del resto de ciudadanos (Martín \& León, 2011)
Las actitudes pueden suponer, para las personas con discapacidad, mayores barreras que las derivadas de su propia situación, influyendo enormemente en su adaptación a los diferentes contextos, tanto académico, social como laboral (Martín \& León, 2011). El modo en que los docentes responden a las necesidades de sus alumnos es una variable mucho más poderosa para determinar el éxito de la integración que cualquier estrategia administrativa o curricular. A través de la actitud positiva del profesorado se favorece que el alumno se sienta valorado en las aulas y que perciba que posee las mismas oportunidades y derechos (Moreno, Rodríguez, Saldaña, \& Aguilera, 2006). Por tanto, los objetivos que han establecido para esta investigación son determinar cual es la percepción de los docentes que mantienen relación con la discapacidad ante las adaptaciones, valorar la percepción de docentes y estudiantes en cuanto a los deberes del profesorado, analizar cual es la realidad de las adaptaciones que se llevan a cabo en las aulas, estudiar la percepción sobre la accesibilidad, analizar el grado de sensibilización de la comunidad universitaria, valorar el grado de conocimientos de los participantes sobre las necesidades de los estudiantes con discapacidad y, por último, determinar si existe asociación entre los años de estudios/experiencia profesional o la rama de conocimiento de los participantes con presentar unas mejores relaciones con los estudiantes con discapacidad.

\section{Participantes}

\section{Método}

Estudio descriptivo de prevalencia en el que la muestra estuvo compuesta por docentes y estudiantes de la Universidad de A Coruña (UDC). Se ha accedido a la muestra a través del Servicio de Atención a la Diversidad (ADI) de la misma universidad. La muestra analizada constaba de 121 docentes y 345 estudiantes.

\section{Instrumentos}

Se han realizado mediciones de las actitudes, a través de la escala "Cuestiones sobre Universidad y Discapacidad" (CUNIDIS) (Figura 1) (Rodríguez Martín \& Álvarez Arregui, 2013), basada en una escala tipo Likert de 5 puntos, compuesta por 40 ítems y estructurada en cuatro bloques de contenido relacionados con los deberes del profesorado, las acciones que el profesorado realiza, la accesibilidad y la sensibilización de la comunidad universitaria. 


\begin{tabular}{|c|c|c|c|c|}
\hline \multicolumn{5}{|c|}{ Final version of the CUNIDIS Scale [in Spanish] } \\
\hline \multicolumn{5}{|c|}{ Indique su grado de acuerdo (de I a 5) con las afirmaciones que se realizan } \\
\hline$I$ & 2 & & 4 & 5 \\
\hline $\begin{array}{c}\text { Totalmente en } \\
\text { desacuerdo }\end{array}$ & $\begin{array}{l}\text { Poco de } \\
\text { acuerdo }\end{array}$ & De acuerdo & $\begin{array}{l}\text { Muy de } \\
\text { acuerdo }\end{array}$ & $\begin{array}{c}\text { Totalmente de } \\
\text { acuerdo }\end{array}$ \\
\hline \multicolumn{5}{|c|}{ Respecto a los estudiantes con discapacidad, el profesorado debe: } \\
\hline \multicolumn{5}{|c|}{ 1. ... adaptar los objetivos de las asignaturas } \\
\hline \multicolumn{5}{|c|}{ 2. ... adaptar los contenidos de las asignaturas } \\
\hline \multicolumn{5}{|c|}{ 3. ... adaptar la metodología empleada en las clases } \\
\hline \multicolumn{5}{|c|}{ 4. ... adaptar las actividades a desarrollar en las asignaturas } \\
\hline \multicolumn{5}{|c|}{ 5. ... adaptar los materiales empleados en las actividades } \\
\hline \multicolumn{5}{|c|}{ 6. .... adaptar los instrumentos de evaluación/ } \\
\hline \multicolumn{5}{|c|}{ 7. ... adaptar los criterios de evaluación/calificación } \\
\hline \multicolumn{5}{|c|}{ 8. .... adaptar las prácticas de la titulación } \\
\hline \multicolumn{5}{|c|}{ 9. a... ampliar el tiempo para exámenes y entregar trabajos } \\
\hline \multicolumn{5}{|c|}{ 10. .... realizar tutorías de manera habitual } \\
\hline Respecto a los & ntes co & tad, en 1 & |el aula & sorado: \\
\hline
\end{tabular}

Figura 1. Escala CUNIDIS

Se han realizado también mediciones relacionadas con las características de los participantes: sociodemográficas, académicas y discapacidad (Tabla $1)$.

Tabla 1.

Mediciones de las características de los participantes

\begin{tabular}{|c|c|}
\hline Docentes & Alumnos \\
\hline \multicolumn{2}{|c|}{ Sociodemográficas } \\
\hline Edad & Edad \\
\hline Sexo & Sexo \\
\hline Estado civil & Estado civil \\
\hline \multicolumn{2}{|c|}{ Académicas } \\
\hline Licenciatura/Grado & Estudios que cursa \\
\hline Facultad donde trabaja & Facultad donde estudia \\
\hline Años de experiencia & Años cursados \\
\hline \multicolumn{2}{|c|}{ Discapacidad } \\
\hline Tener discapacidad & Tener discapacidad \\
\hline $\begin{array}{c}\text { Relación con } \\
\text { discapacidad }\end{array}$ & $\begin{array}{cc}\text { Relación } & \text { con } \\
\text { discapacidad } & \end{array}$ \\
\hline
\end{tabular}

Las mediciones expuestas se han unificado en una encuesta online, autoadministrada y anonimizada.

\section{Procedimiento}

Se ha realizado un estudio descriptivo de las variables incluidas en el estudio. Para determinar la asociación entre variables cualitativas se ha utilizado el estadístico chi cuadrado y para comparar medias un análisis de varianza (ANOVA). Para determinar asociaciones con las variables experiencia y profesión se ha realizado un análisis multivariado de regresión logística en el que la variable dependiente ha sido la agrupación de las respuestas a los ítems en dos categorías (desacuerdo/acuerdo).

\section{Resultados}

El análisis descriptivo de las variables indica la existencia de un $79,9 \%$ de participantes que poseen algún tipo de relación con la discapacidad, de éstos la mayoría $(19,4 \%)$ indican que se trata de una relación de amistad. El 62,9\% de los participantes son mujeres y el $36,8 \%$ hombres. En cuanto a la rama de conocimiento, el $44,2 \%$ pertenecen a la rama de Ciencias Sociales y Jurídicas y el $30 \%$ a la de Ingeniería y Arquitectura. Han afirmado tener discapacidad el $3,8 \%$ de los encuestados. La media de edad se ha situado en 29,3 años y la mitad de los docentes poseen 17 años de experiencia profesional. En el análisis descriptivo de la encuesta, los datos más destacables son los siguientes:

- $\quad$ El 52,8\% de los participantes están totalmente de acuerdo con la necesidad de adaptar los materiales utilizados durante la docencia, mientras que únicamente el $25,1 \%$ están totalmente de acuerdo con adaptar también los criterios de evaluación y cualificación.

- $\quad$ El 24,7\% de los encuestados indicaron que en la actualidad no se están adaptando los contenidos de las asignaturas en la realidad del aula.

- El 43,2\% de los participantes creen que los estudiantes con discapacidad participan en todas las actividades que se proponen en las aulas.

- $\quad$ El 74,6\% de los encuestados creen que un estudiante con discapacidad puede ser un buen profesional.

Por otro lado, en base a los objetivos establecidos para la investigación los principales datos obtenidos son los siguientes:

- $\quad$ El 57,3\% de los docentes que tienen relación con la discapacidad no adaptan los contenidos de las materias.

- $\quad$ Sólo el 25,9\% de los docentes están de acuerdo con que los materiales impresos o audiovisuales que se entregan en las aulas están adaptados.

- El 49,5\% están en desacuerdo con que las personas con discapacidad cuentan con los apoyos humanos y materiales para seguir las clases.

- $\quad$ El $65,1 \%$ de los docentes están en desacuerdo con que el profesorado de la universidad está formado en temas relacionados con la discapacidad, el alumnado está de acuerdo en un 45,8\%.

- $\quad$ El 74,8\% de los docentes y el 74,5\% de los estudiantes están totalmente de acuerdo con que un estudiante con discapacidad puede ser un buen profesional.

Se ha encontrado una asociación estadísticamente significativa entre los años de estudios universitarios cursados y la relación con los estudiantes con discapacidad $(\mathrm{p}=0,042)$, pero no se ha podido determinar si más o menos años implican mejores o peores relaciones con la discapacidad. No existe relación entre los años de experiencia docente y la relación con la discapacidad. No se ha podido establecer relación entre la rama de conocimiento y la relación con la discapacidad. 


\section{Discusión}

La muestra de este estudio ha contado con 121 docentes y 345 estudiantes de la Universidad de A Coruña. De la totalidad de los participantes, el 3,8\% afirma tener discapacidad. En este ámbito, es de reseñar que este no es el primer estudio realizado en la Universidad de A Coruña, ya que Novo-Corti, MuñozCantero y Calvo-Porral (2011) analizaron una muestra de 180 estudiantes de las diferentes ramas de Economía y Empresa, de los que únicamente el 0,62\% padecían algún tipo de discapacidad.

\section{Actitudes hacia la adaptación de las materias}

Este bloque de contenido aborda los ítems relacionados con los deberes que tienen los docentes para con los estudiantes con discapacidad en el ámbito universitario. En conjunto, el $52,8 \%$ de los participantes, indican que es necesario adaptar los materiales empleados en las actividades docentes, mientras que sólo el $25,1 \%$ señalan que es preciso adaptar los criterios de evaluación y de cualificación para los alumnos con discapacidad en base a sus necesidades. Cuando se estratifican las respuestas por profesorado y alumnado, el $41 \%$ de los docentes creen que no es preciso adaptar los criterios de evaluación y cualificación para las personas con discapacidad, mientras que los estudiantes apoyan esta idea sólo en un $30,8 \%$.

Estos resultados no son del todo coincidentes con los datos publicados por Rodríguez-Martín y ÁlvarezArregui (2015), ya que si bien en su trabajo, como en el nuestro, los estudiantes presentan respuestas favorables hacia la adaptación de las actividades, de los materiales empleados y de las prácticas, la mayoría de los docentes, por el contrario, consideraban que no deben adaptar los objetivos, los contenidos, los instrumentos ni los criterios de evaluación. Esta diferencia, pensamos, puede deberse a que la adaptación de estos criterios a favor de los estudiantes con discapacidad puede entenderse por el profesorado universitario como un trato de favor hacia este colectivo, coincidiendo en este sentido con las reflexiones del estudio publicado por Moriña Díez, López Gavira, Melero Aguilar, Cortés Vega y Molina Romo (2013) en el que, además, se viene a decir que la actitud de los docentes no siempre es todo lo adecuada que debería ante las adaptaciones.

La actitud favorable del colectivo de alumnos hacia la adaptación de las diversas acciones docentes para las personas con discapacidad no es nada nuevo. Ya en el año 2011, en el trabajo publicado por Novo-Corti, Muñoz-Cantero y Calvo-Porral (2011), los estudiantes manifestaron que asumen la necesidad de políticas inclusivas, por lo que las actuaciones y adaptaciones bajo apoyo institucional serían aceptadas con agrado; sin embargo, en cuanto a la promoción de la inclusión de las personas con discapacidad, los estudiantes participantes del estudio manifestaron que prefieren que esta labor recaiga sobre las instituciones públicas.

Actitudes hacia la acción docente que el profesorado realiza
Con respecto a las acciones levadas a cabo a favor de los estudiantes con discapacidad por los docentes en la realidad del aula, el $25 \%$ de los participantes indicaron que en la actualidad no se llevan a cabo las adaptaciones correspondientes a los contenidos de las materias que se imparten para los estudiantes con discapacidad.

Sin embargo, al realizar la estratificación por docentes, más de la mitad de estos $(58,5 \%)$ indicaron que no adaptan los contenidos de las materias que imparten para las personas con discapacidad. Siguiendo la misma línea que en nuestro estudio, los docentes encuestados por Rodríguez-Martín y Álvarez-Arregui (2015) también mencionaron que en la práctica real del aula no se adaptan los objetivos $(79,7 \%)$, los contenidos $(80,5 \%)$ ni los instrumentos y criterios de evaluación $(67,3 \%)$, mientras que los estudiantes coinciden con la tendencia del profesorado, identificando que no se realiza la adaptación de los objetivos $(36,8 \%)$ ni de los contenidos $(39,6 \%)$ de las diferentes materias.

En otro estudio llevado a cabo en el ámbito universitario por Moriña-Diez, López Gavira, Melero Aguilar, Cortés Vega y Molina Romo (2013), los participantes afirmaron que el profesorado no se muestra predispuesto a facilitar, por adelantado, el material docente que se va a utilizar para la docencia. En la misma línea también identificaron la falta de previsión de los docentes para la adaptación de los recursos y materiales con el tiempo necesario.

En base al objetivo general de esta investigación se detectó que el $57 \%$ de los docentes encuestados que tienen relación con alguna persona con discapacidad indicó que, a diario en su labor profesional, no adapta los contenidos de las materias para este colectivo. Los resultados del estudio de Rodríguez-Martín y ÁlvarezArregui (2015), apoyan los de nuestra investigación, ya que el profesorado y los estudiantes consideran que en la práctica real del aula no se adaptan los contenidos de las diferentes materias que se imparten durante la docencia.

\section{Actitudes sobre la accesibilidad de los estudiantes con discapacidad en la comunidad universitaria}

En cuanto a los ítems relacionados con la accesibilidad de los estudiantes con discapacidad de la UDC, menos de la mitad de los docentes y estudiantes consideran que los estudiantes con discapacidad participan en todas las actividades que se proponen de manera cotidiana en el aula. Esto pone de manifiesto que la inclusión de las personas con discapacidad en las aulas no es totalmente real, ya que no todos los estudiantes participan de la misma manera durante la docencia, hecho que puede estar influenciado por la existencia de diversas barreras arquitectónicas, de la comunicación, actitudinales, sociales o culturales.

Sin embargo, en cuanto a la accesibilidad de los materiales que se aportan en la docencia y en las actividades universitarias, poco más de la mitad de los participantes $(50,8 \%)$ consideró que los materiales impresos o audiovisuales que se entregan para llevar a cabo la docencia se encuentran adaptados para las personas con discapacidad. De nuevo esto parece indicar que es preciso garantizar la existencia de una 
formación en accesibilidad educativa dirigida a los docentes, que permita que los materiales empleados por éstos puedan ser accesibles para todos los estudiantes, ya que la otra mitad de los participantes opinan que no están adaptados. Este dato se encuentra apoyado por los resultados obtenidos en la investigación de RodríguezMartín y Álvarez-Arregui (2015) en la que, tanto el profesorado $(48,9 \%)$ como los estudiantes (57\%) reconocen que se adaptan algunos materiales y se emplean tecnologías de apoyo para el seguimiento de las clases por parte de los estudiantes con discapacidad.

Actitudes sobre las relaciones sociales y la sensibilización de la comunidad universitaria hacia los estudiantes con discapacidad

En los resultados se evidenció que el $75 \%$ de los participantes de este estudio consideran que un estudiante con discapacidad puede ser un buen profesional y desarrollar su labor de manera satisfactoria. Esto parece indicar una actitud positiva hacia la inclusión educativa, y posteriormente laboral, de los estudiantes con discapacidad, ya que también se ha encontrado en el estudio de Rodríguez-Martín y Álvarez-Arregui (2015) que mientras que el profesorado considera que los estudiantes con discapacidad no pueden estudiar cualquier titulación (62\%), los estudiantes tienen una actitud favorable en ese sentido $(77,8 \%)$. Por tanto, puede observarse que, a pesar de la multitud de creencias y prejuicios que puedan existir ante las personas con discapacidad, actualmente estas actitudes están cambiando.

Sin embargo, uno de los datos más relevantes que se extrae del presente estudio fue que los estudiantes indicaron que el profesorado no posee los conocimientos precisos para dar una respuesta adecuada a las necesidades de los estudiantes con discapacidad, información corroborada por los propios docentes. Esta información coincide con la de los estudios de Rodríguez-Martín y Álvarez-Arregui (2015) y de AbuHamour (2013), en los que los participantes, docentes y estudiantes, consideran que el profesorado no está formado para dar respuesta a los estudiantes con discapacidad.

\section{Asociación entre los años de experiencia y la relación con la discapacidad}

Se ha intentado determinar si existe algún tipo de asociación entre los años de experiencia docente y de años de estudios cursados por los alumnos, con presentar unas mejores relaciones con las personas con discapacidad. En cuanto a los años de estudios cursados por los estudiantes, se ha encontrado una asociación estadísticamente significativa, que permite determinar que los años de estudios cursados repercuten en la relación que tienen estos estudiantes con los estudiantes con discapacidad. No se puede determinar si a mayor número de años de estudios cursados por los estudiantes las relaciones son mejores o peores.

En la actualidad existen diferencias relevantes entre los resultados obtenidos en diferentes estudios, por ejemplo, Suriá Martínez (2011) en su estudio en la Universidad de Alicante detectó diferencias estadísticamente significativas en las actitudes de los estudiantes en base al curso en el que desarrollan sus estudios. Sin embargo existen discrepancias entre sí a mayor o menor experiencia de estudios cursados repercute en unas mejores o peores relaciones puesto que Abu-Hamour (2013) en su estudio, llevado a cabo en Jordania, detectó que los miembros de la facultad con menos años de experiencia presentan actitudes más positivas hacia los estudiantes con discapacidad; mientras que Alonso Olea, Navarro y Vicente (2007) determinaron que en cuanto al nivel de estudios cursado, existen diferencias significativas, presentando actitudes más positivas los estudiantes de último curso.

\section{Asociación entre la rama de conocimiento y la relación con la discapacidad}

Por último se ha intentado determinar si existe algún tipo de asociación estadística entre la rama de conocimiento en la que docentes y estudiantes desarrollan su labor profesional y estudiantil, respectivamente, con presentar unas mejores relaciones con las personas con discapacidad; sin embargo no se puede establecer dicha asociación debido al pequeño tamaño de la muestra en algunas de las categorías del estudio.

Sin embargo en el estudio de Rodríguez-Martín y Álvarez-Arregui (2015) se detectó que profesorado y estudiantes de la rama de Ciencias Sociales y Jurídicas, y en menor medida los adscritos a Ciencias de la Salud, tienen puntuaciones superiores y más favorables hacia las cuestiones presentadas con respecto a los de la rama de Arquitectura e Ingenierías. Esto puede ser debido a los contenidos que se desarrollan en las antedichas ramas de conocimiento, ya que en las ramas Sociales, Jurídicas y en las de Ciencias de la Salud, el conocimiento de la discapacidad es un contenido a tratar en diversas materias, en mayor medida que en las ramas de Arquitectura o en las Ingenierías, a pesar de la importancia con la que cuenta el conocimiento de la discapacidad en el ámbito de la Arquitectura, ya que ayudaría a garantizar la accesibilidad universal.

\section{Conclusiones}

1. La mayoría de los participantes indicaron que es necesario adaptar los materiales empleados en las actividades que se desarrollan en las aulas universitarias. Tres de cada cuatro encuestados creen que no es preciso adaptar los criterios de evaluación y de cualificación para los alumnos con discapacidad.

2. Sólo una minoría indica que no se realizan adaptaciones para las materias que se imparten para los estudiantes universitarios con discapacidad.

3. Más de la mitad de los participantes indican que los estudiantes con discapacidad no participan en todas las actividades que se llevan a cabo durante la docencia.

4. La mayoría de los encuestados creen que un estudiante universitario con discapacidad puede ser un buen profesional el día de mañana.

5. Más de la mitad de los docentes indicaron que no adaptan los contenidos de las materias para las 
personas con discapacidad ni los materiales impresos y audiovisuales que se entregan en las aulas.

6. Una mayoría de los docentes refirieron que el profesorado universitario no está formado para dar respuesta a las necesidades de los estudiantes con discapacidad, y la mitad de los estudiantes apoyan esta opinión.

7. Existe relación entre los años de estudios cursados por el alumnado y la relación que mantienen con los estudiantes con discapacidad.

8. En base a los resultados obtenidos, creemos que los estudiantes y docentes de la UDC presentan, de manera general, unas actitudes favorables hacia la inclusión de los estudiantes con discapacidad.

\section{Referencias}

Abu-Hamour, B. (2013). Faculty attitudes toward students with disabilities in a public university in Jordan. International Education Studies , 6 (12), 74.

Alonso Olea, M., Navarro, R., \& Vicente, L. (2007). Actitudes hacia la diversidad en estudiantes universitarios. Fòrum de recerca (13).

Fundación Universia, \& Cermi. (2013). II Estudio sobre el grado de inclusión del sistema universitario español respecto de la realidad de la discapacidad. Obtenido de estudiosobregradodeinclusion_accesible.pdf

Martín, M., \& León, M. (2011). Los docentes de la universidad de Burgos y su actitud hacia las personas con discapacidad. Siglo Cero: Revista Española sobre Discapacidad Intelectual , 42 (240), 50-78.
Martínez, R., Bueno, A., \& Limiñana, A. (2011). Prejuicios entre los estudiantes hacia las personas con discapacidad: Reflexiones a partir del caso de la universidad de Alicante. Alternativas: cuadernos de trabajo social , 25 (3), 370-6.

Moreno, F., Rodríguez, I., Saldaña, D., \& Aguilera, A. (2006). Actitudes ante la discapacidad en el alumando universitario matriculado en materias afines. RIE , 40 (5).

Moriña Díez, A., López Gavira, R., Melero Aguilar, N., Cortés Vega, M., \& Molina Romo, V. (2013). El profesorado en la universidad ante el alumnado con discapacidad ¿Tendiendo puentes o levantando muros? Revista de docencia universitaria , 11 (3), 423-442.

Novo-Corti, I., Muñoz-Cantero, J., \& Calvo-Porral, C. (2011). Análisis de las actitudes de los jóvenes universitarios hacia la discapacidad: Un enfoque desde la teoría de la acción razonada. RELIEVE , 17 (2).

Rodríguez Martín, A., \& Álvarez Arregui, E. (2013). Development and validation of a scale to identify attitudes toward disability in higher education. Psicothema Revista de Psicología , 25 (3), 370-6.

Rodríguez-Martín, A., \& Álvarez-Arregui, E. (2015). Universidad y discapacidad. Actitudes del profesorado y de estudiantes. Perfiles educativos , 37 (147).

Suriá Martínez, R. (2011). Análisis comparativo sobre las actitudes de los estudiantes hacia sus compañeros con discapacidad. Electronic journal of research in educational psychology, 9 (23), 197-219. 